Check for updates

Cite this: Mater. Adv., 2022, 3,3526

Received 17th January 2022, Accepted 3rd March 2022

DOI: $10.1039 / \mathrm{d} 2 \mathrm{ma} 00048 \mathrm{~b}$

rsc.li/materials-advances

\section{DFT and TD-DFT studies of new triphenylamine-based (D-A-D) donor materials for high-efficiency organic solar cells $\dagger$}

\begin{abstract}
Numbury Surendra Babu
The DFT approach of Becke's three-parameter compound (B3LYP) was employed in all studies of triphenylamine (TPA) molecules as a donor (D) for solar cell applications in this work. We created three donor molecules (D-A-D) with a strong donor moiety of triphenylamine (TPA) connected to three distinct acceptor (A) units, including benzo[c][1,2,5]thiadiazole, [1,2,5]thiadiazolo[3,4-c]pyridine, and $[1,2,5]$ thiadiazolo[3,4-d]pyridazine. DFT and TD-DFT methods were used to examine the photophysical properties, frontier molecular orbitals (FMOs), density of states (DOS), and open-circuit voltages $\left(V_{O C}\right)$. The designed molecule D3 has been proven to be the best candidate for solar cell applications over the others due to its promising photovoltaic properties, which include the lowest bandgap (1.53 eV) and excitation energy $(1.21 \mathrm{eV})$, a low binding energy $\left(E_{\mathrm{b}}=0.31 \mathrm{eV}\right)$, and the highest max values of $964.01 \mathrm{~nm}$ and $1017.00 \mathrm{~nm}$ in the gas and solvent phases, respectively. This theoretically developed model indicates that $D-A-D$ unit modifications are a viable alternative to achieve the desired optoelectronic properties. As a result, the developed D1, D2, and D3 molecules are exceptional and strongly suggested to experimentalists for the future creation of highly efficient solar cell systems.
\end{abstract}

\section{Introduction}

In recent years, depleting energy resources have necessitated a favourable alternative that might assist us in producing energy at a lower cost and with fewer environmental concerns (i.e. pollution, greenhouse effect, etc.). To safeguard the environment from climate change, efforts are being made to develop environmentally friendly energy options. Renewable energy is also known as fossil fuel recycling, and its sources are expected never to run out, just as sustainable energy sources will never run out. ${ }^{1}$ Solar energy, ${ }^{2}$ wind energy, ${ }^{3}$ hydropower energy, ${ }^{4}$ biogas energy, ${ }^{5}$ and tidal energy ${ }^{6}$ are high-potential renewable sources. Sunlight is a common bestowal that provides inexpensive and environmentally friendly energy. Photovoltaic devices and solar cells that work on the photoemission phenomenon convert sunlight into electricity. ${ }^{7,8}$ The acceptor sections of a photovoltaic cell capture sunlight and convert it into electricity. ${ }^{9}$ The donor, acceptor, and bridge are the three essential components of a photovoltaic solar cell. The donor portion sends electrons to the acceptor, which uses electrons to capture sunlight, and the bridge works as a spacer between the acceptor and donor

Computational Quantum Chemistry Lab, Department of Chemistry, College of Natural and Mathematical Science, The University of Dodoma, Dodoma, Tanzania. E-mail: nsbabusk@gmail.com

$\dagger$ Electronic supplementary information (ESI) available. See DOI: 10.1039/d2ma00048b parts. ${ }^{10}$ Fundamentally, there are three types of photovoltaic solar cells: (1) inorganic solar cells with silicon semiconducting materials, (2) organic solar cells with polymer and tiny acceptor moieties, and (3) hybrid solar cells (combined organic and inorganic materials). ${ }^{11}$ Photovoltaic devices used silicon as the semiconducting material in early investigations, now accessible on the market. With a maximum power conversion efficiency (PCE), silicon-based photovoltaic solar cells exhibited reduced toxicity, high abundance, high efficiency, and high thermal security, ${ }^{12}$ but they had certain disadvantages such as rigidity, high price, and non-tunable energy levels. Because of the drawbacks of silicon-based solar cells, they are no longer being considered.

A low-cost, lightweight, flexible organic photovoltaic (OPV) cell has the potential to deliver solar energy to a variety of intriguing applications. The introduction of a bulk heterojunction (BHJ) architecture, in which push-pull (or D-A) conjugated copolymer donors (absorbing the solar spectrum in the near-IR region of 680-950 $\mathrm{nm}$ ) form an interpenetrating network with fullerene-derivative acceptors, improved the power conversion efficiency (PCE) by 12\%. For high PCE OPV donors, a narrow bandgap, high charge carrier mobility and efficient charge separation and collection are desirable and are frequently achieved through morphological control.

Because of their homogeneous and well-defined molecular structure, OPV cells with tiny molecular donors have recently 
gained much attention because they avoid batch-to-batch changes in the molecular weight, polydispersity, and purity of polymeric donors.

Small-molecule donors could provide more consistent morphological control and finer energy-level control through chemical-structure modifications. ${ }^{13}$

When they were first introduced in 2006, they attracted little attention because of their extremely low PCE (1\%), but in $2012^{13}$ the PCE improved to 6.7 percent with a D1-A-D2-A-D1 type of small-molecule donor, DTS(PTTh2)2, and it has since improved to 8-9 percent with continuous optimization of the molecular structure that modifies or replaces each unit to introduce new A-D-A combinations. ${ }^{14-17}$

The creation of current donor-acceptor (D-A) small molecules with a reduced energy gap $\left(E_{\mathrm{g}}\right)$ between HOMOs and LUMOs $^{18}$ due to a fast electron transfer between electrondonating and electron-withdrawing moieties ${ }^{19}$ is an essential strategy for improving the performance of solar systems. Nowadays, DFT-based molecular engineering has allowed us to design new small molecular donor materials and theoretically test their photovoltaic and optoelectronic capabilities, which can then be compared to empirically manufactured molecules. Furthermore, scientists can investigate the many efficiency parameters of photovoltaic materials using computer analysis. $^{20-22}$

TPA-based materials have excellent electro-optical characteristics and improve the material hole mobility. ${ }^{10}$ In addition, the terminal groups of TPA are substituted with new highly efficient groups with strong electron-withdrawing properties in these developed structures. Photophysical characteristics, frontier molecular orbitals (FMOs), reorganization energies, density of states (DOS) and overlap population density of states (OPDOS), open-circuit voltages $\left(V_{\mathrm{OC}}\right)$, transition density matrix (TDMs), and surface and charge transfer analysis were all assessed using the DFT and TD-DFT approaches. The results indicated that DFT-based small-molecule donors (D1-D4) are superior for high-efficiency solar cells.

We created three donor molecules (D-A-D) with a strong triphenylamine (TPA) donor moiety coupled to three distinct acceptor (A) units, including benzo $[c][1,2,5]$ thiadiazole, $[1,2,5]$ thiadiazolo[3,4-c]pyridine and $[1,2,5]$ thiadiazolo[3,4- $d]$ pyridazine (Scheme 1).

\section{Computational details}

The goal of this study is to improve the optoelectronic capabilities of triphenylamine based donor molecules using specific acceptor units benzo[c][1,2,5]thiadiazole (D1), $[1,2,5]$ thiadiazolo[3,4-c]pyridine (D2), and $[1,2,5]$ thiadiazolo[3,4- $d]$ pyridazine(D3); the structural modification of the donor molecules is done by keeping the acceptors (central units) (Scheme 1). The Gaussian 09 suite $^{23}$ was used for all the calculations. The GaussView 5.0.8 program $^{24}$ was used to visualize the results and draw the structures. The geometries and electronic characteristics of the molecules were investigated using density
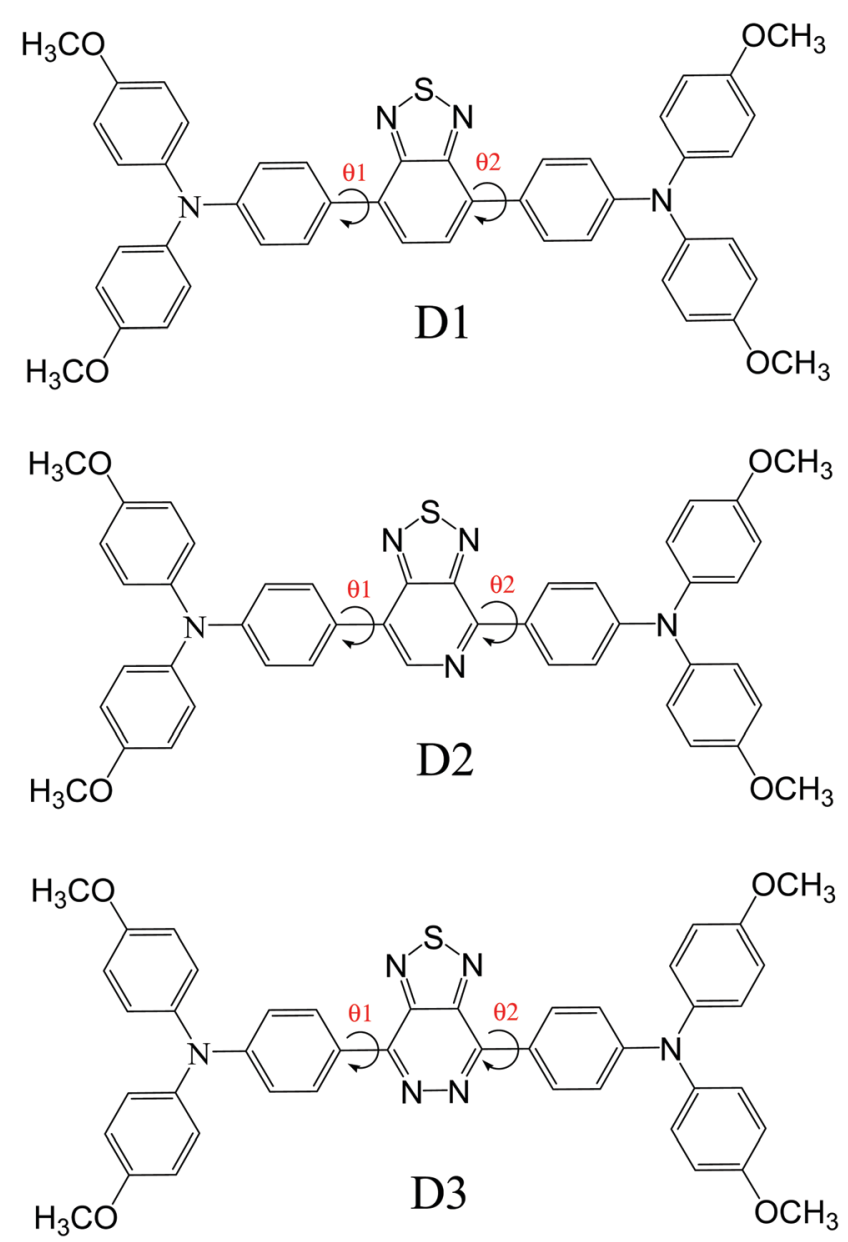

Scheme 1 Structures of the studied D-A-D molecules with D1, D2 and D3.

functional theory (DFT) methods. ${ }^{25-27}$ The B3LYP ${ }^{28}$ method of DFT with the $6-31 \mathrm{G}^{29}$ basis set was used to optimize the geometries of all molecules (neutral, anionic, and cationic states). In the gas phase, the excited state geometries were computed using TD-DFT at the TD-B3LYP/6-31G $\mathrm{G}^{20-32}$ level of theories. The polarization continuum model $(\mathrm{PCM})^{33}$ was used to investigate the solvent effect. The density of states (DOS) spectra were depicted using Gauss Sum 2.2.0. ${ }^{34}$

\section{Results and discussion}

\subsection{Optimized geometries}

In the visible light absorption of a chemical substance, planarity in molecular geometry and the related-electron conjugation over the backbone are crucial. Fig. 1 (Fig. S1, ESI $\dagger-$ in chlorobenzene) shows the optimized molecular structures of D1, D2, and D3. The D-A-D has planarized the geometry of the resultant polymer by producing a delocalized-electronic cloud density across the backbone, as shown by a perfect $180^{\circ}$ dihedral (Table 1) of the $\mathrm{D}-\mathrm{A}-\mathrm{D}$. The low cost and uncomplicated synthesis technique of $\mathrm{D}-\mathrm{A}-\mathrm{D}$ can be attributed to its intra-chain dihedral angle $\left(180^{\circ}\right)$. This claim also contradicts the current research. ${ }^{35-37}$ 


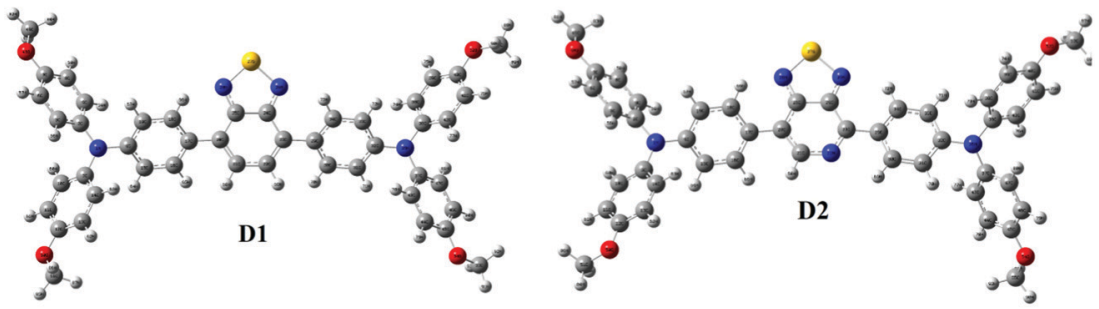

Fig. 1 Optimized geometric structures of the designed donors (D1, D2 and D3).

Table 1 Dihedral angles between the neighbouring rings of $D-A-D$ molecules using the DFT/B3LYP/6-31G method

\begin{tabular}{|c|c|c|c|c|c|c|}
\hline \multirow[b]{2}{*}{ Donor } & \multicolumn{2}{|l|}{ Gas } & \multicolumn{2}{|c|}{ Chlorobenzene } & \multirow{2}{*}{$\frac{\text { Gas }}{\mu_{\mathrm{e}} \text { (Debye) }}$} & \multirow{2}{*}{$\frac{\text { Chlorobenzene }}{\mu_{\mathrm{e}} \text { (Debye) }}$} \\
\hline & $\theta 1$ & $\theta 2$ & $\theta 1$ & $\theta 2$ & & \\
\hline D1 & 148.96 & 148.91 & 147.39 & 147.33 & 4.3873 & 5.5353 \\
\hline D2 & 150.03 & 177.42 & 148.25 & 178.29 & 4.5499 & 5.6140 \\
\hline D3 & 178.12 & 177.17 & 178.26 & 177.01 & 3.0075 & 3.9134 \\
\hline
\end{tabular}

Donor dihedral angles are also close to planarity in intramolecular rings. The internal steric hindrance of the $\mathrm{N}$ atoms of adjacent rings of benzothiadiazole caused them to fall out of the plane as well, causing A to deviate from $180^{\circ}$.

\subsection{Frontier molecular orbitals and electronic properties}

Studies of frontier molecular orbitals are used to determine the excited-state features of computationally created molecules for organic solar cells (OSCs) (FMOs). The charge transport mechanism was better understood because of the excited-state features of organic solar cells. By generating effective OSC devices, the photovoltaic efficiency of OSCs is increased through alteration of the charge transfer mechanism within molecules. Changing the charge transfer mechanism in OSCs made with a tiny molecule necessitates energy. The energy gap $\left(E_{\mathrm{g}}\right)$ becomes minimal when manufacturing tiny moleculebased OSCs. The photovoltaic performance of OSCs will be better if the $E_{\mathrm{g}}$ value is lower and vice versa. ${ }^{20}$

The HOMO and LUMO describe the bonding and antibonding properties of designer molecules in their excited and ground states, respectively. The charge transfer in OSCs is accomplished from the donor HOMO to the acceptor LUMO and vice versa for the current generation. The efficiency of cells, the amount of current, and the voltages are all directly related to electron transfer. As a result, the phenomenon of electron excitation is improved, which improves the electron transport. ${ }^{38}$ The stability and testability of HOMO-LUMO energy orbitals also play a role in electronic excitation. More significant electronic excitation follows more de-stable filled highest occupied molecular orbitals (HOMOs) and vice versa. ${ }^{39}$ However, to capture more sunlight and ensure good device performance, the energy of LUMOs should be increased. ${ }^{40}$ Highly stable LUMOs can keep the maximum number of electrons in the acceptor region of the cell for extended periods, allowing more sunlight to be absorbed and converted into electricity, boosting the device performance.

Fig. 2 shows the FMO distribution patterns for D1, D2, and D3 at B3LYP/6-31G. For donor molecules, the HOMO-LUMO distribution is nearly identical. The HOMO is found on the TPA moiety in all compounds, but the LUMO is distributed throughout the molecule with the highest density on the central acceptor unit. The methoxy group enhances the energies of the HOMO and LUMO in D2 and D3, but the effect is more

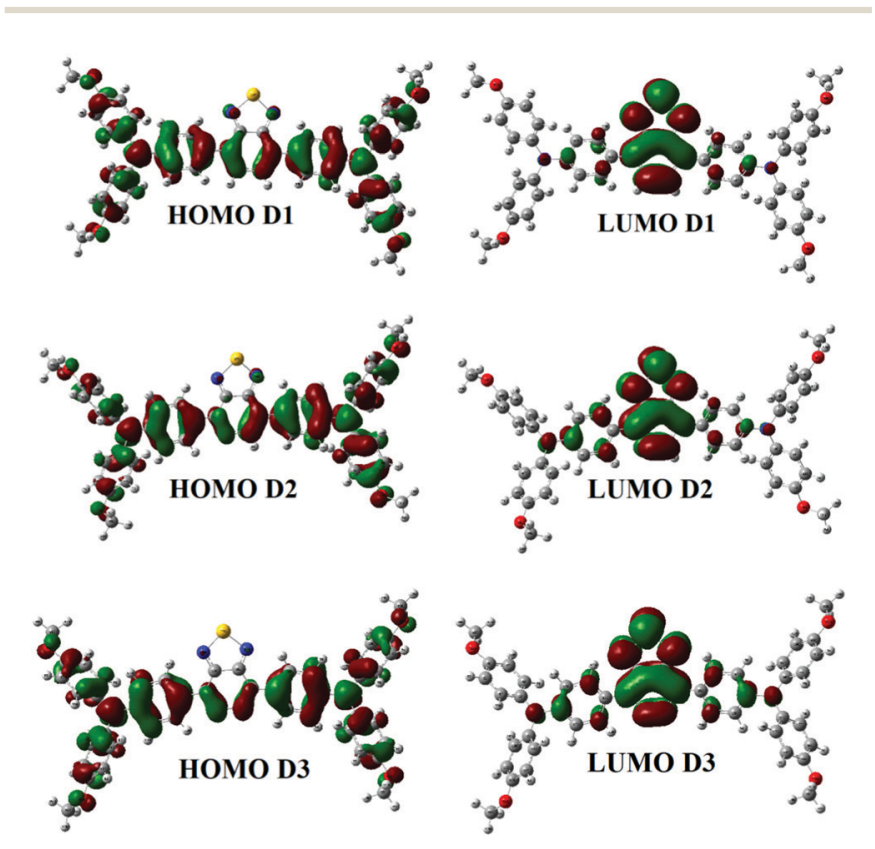

Fig. 2 Contours of the HOMO and LUMO of D1, D2 and D3 in the gas phase. 
potent in D1. Therefore, an increase in the energy level is expected for electron-donating methoxy groups. The methoxy groups in D3 have a similar impact on the HOMO but have the opposite effect on the LUMO (which is more stabilized than D1).

We see a relatively uniform electron distribution for D3 in the HOMO (Fig. S2, ESI $\dagger$ - in chlorobenzene), implying that the hole density will be evenly distributed in the electronically excited molecule and the charge-separated hole carrier. The LUMO is found on the BT and CMA moieties of the molecules, which is consistent with the projected internal acceptor capability of this unit. Due to the total delocalization of donor moieties like TPA and methoxy $\left(\mathrm{OCH}_{3}\right)$ groups, the charge density for HOMO 1 in D1-D3 is dispersed over the entire molecule, while for LUMO+1, the density (in all compounds) is directed towards the acceptor unit of the molecule (away from the donor side). The electron density shifts towards TPA rings in the case of the HOMO. There is a variation in all compounds for HOMO 3. The electron density in HOMO 3 is pushed towards the TPA ring in M1, while it is distributed across the entire molecule in D2 and D3. In LUMO+2, the electron density is distributed across the whole molecule (in all the compounds), whereas, in LUMO+3, the electron density moves towards acceptor units (M1 and M3). The electron density in LUMO+3 is concentrated on the TPA portion for D2 (Fig. S3, ESI $\dagger$ ).

At the B3LYP/6-31G level of DFT, the HOMO and LUMO energy values for D1, D2, and D3 molecules are $-4.53591 \mathrm{eV}$, $-4.5691 \mathrm{eV}$ and -4.63631 , and $-2.49488 \mathrm{eV},-2.80127 \mathrm{eV}$, and $-3.08235 \mathrm{eV}$, respectively, as illustrated in Fig. 3 and Table 2. All the developed molecules D1, D2, and D3 had $E_{\mathrm{g}}$ values of $2.0410 \mathrm{eV}, 1.7678 \mathrm{eV}$, and $1.5539 \mathrm{eV}$, respectively. Compared to D1 and D2, the D3 molecule has a lower HOMO value $(-4.636 \mathrm{eV})$. The inclusion of the $[1,2,5]$ thiadiazolo $[3,4-d]$ pyridazine acceptor group gives molecule D3 a higher HOMO value. Because of its lower HOMO value $(4.535 \mathrm{eV})$, the D1 molecule is more stable than the others. The LUMO values of the D1 and D2 molecules are $-2.49488 \mathrm{eV}$ and $-2.80127 \mathrm{eV}$, respectively. D1 $>\mathrm{D} 2>\mathrm{D} 3$ is the decreasing order of $E_{\mathrm{g}}$ (HOMO-LUMO energy gap) of D1-D3 molecules. The most significant charge transfer phenomena are indicated by a small range of $E_{\mathrm{g}}$ in all molecules (D1-D3). The D3 molecule has the lowest $E_{\mathrm{g}}$ value $(1.553 \mathrm{eV})$ compared to the others (D1 and D2), indicating that electrons are transferred from the donor HOMO
Table 2 HOMO-LUMO energy gaps $\left(E_{\mathrm{g}}\right)$, first singlet excitation energies $\left(E_{\mathrm{opt}}\right)$ and exciton binding energies $\left(E_{\mathrm{b}}\right)$

\begin{tabular}{lllllll}
\hline Donor & HOMO & LUMO & $E_{\mathrm{g}}(\mathrm{eV})$ & $E_{\text {Opt }}(\mathrm{eV})$ & $E_{\mathrm{b}}(\mathrm{eV})$ & $V_{\mathrm{OC}}$ \\
\hline Gas & & & & & & \\
D1 & -4.5359 & -2.4948 & 2.0410 & 1.6792 & 0.3618 & 0.5359 \\
D2 & -4.5691 & -2.8012 & 1.7678 & 1.4550 & 0.3128 & 0.5691 \\
D3 & -4.6363 & -3.0823 & 1.5539 & 1.2863 & 0.2676 & 0.6363 \\
Chlorobenzene & & & & & \\
D1 & -2.7155 & 1.9895 & 1.9895 & 1.5947 & 0.3948 & 0.7051 \\
D2 & -3.0301 & 1.7169 & 1.7169 & 1.3656 & 0.3513 & 0.7470 \\
D3 & -3.2915 & 1.5321 & 1.5321 & 1.2193 & 0.3128 & 0.8237
\end{tabular}

(D3) to the acceptor LUMO (D2) and vice versa. Fig. 1 depicts the comparison of the HOMO-LUMO energies and $E_{\mathrm{g}}$. The higher the open-circuit voltage $\left(V_{\mathrm{OC}}\right)$ of the OSCs, the lower the HOMO values of the molecules. ${ }^{41}$

\subsection{Optical properties}

The UV/visible absorption spectra of the suggested photovoltaic materials are simulated using the TD-DFT/B3LYP/6-31G method in both the gas (vacuum) and solvent (chloroform) phases (D1-D3). In both gas and solvent phases, Table 3 shows the values for the maximum absorption wavelength (max), excitation energy $\left(E_{\mathrm{x}}\right)$, oscillator strength $\left(f_{\mathrm{os}}\right)$, and significant $\mathrm{MO}$ assignments. Because of the replacement of different acceptors, the absorption spectra of D1-D3 molecules have been redshifted. As a result, the developed compounds (D1-D3) have max values ranging from 738.46 to $1017.00 \mathrm{~nm}$.

The computed maximum values of D1-D3 molecules with the acceptor benzo $[c][1,2,5]$ thiadiazole in $\mathrm{D} 1,[1,2,5]$ thiadiazolo[3,4-c]pyridine in $\mathrm{D} 2$, and $[1,2,5]$ thiadiazolo[3,4- $d]$ pyridazine in D3 display high, sharp, and broad absorption peaks in the spectra. The quality of creating photovoltaic materials is improved by the broad and intense adsorption characteristics of the molecules. ${ }^{42}$ In a vacuum, the $\lambda_{\max }$ values for D1, D2, and D3 are $738.46 \mathrm{~nm}$, $852.24 \mathrm{~nm}$, and $964.01 \mathrm{~nm}$, and in the chloroform solvent they are $777.58 \mathrm{~nm}, 908.01 \mathrm{~nm}$, and $1017.00 \mathrm{~nm}$, respectively. The efficiency of acceptor units for the designed D1-D3 molecules is described by these near-infrared (NIR) absorption peaks. The D3 molecule has the highest near infrared (NIR) max value in a vacuum and in the chloroform solvent. D3 exhibits a redshift at its maximum due to protracted conjugation and the high charge transfer capacity of the acceptor unit
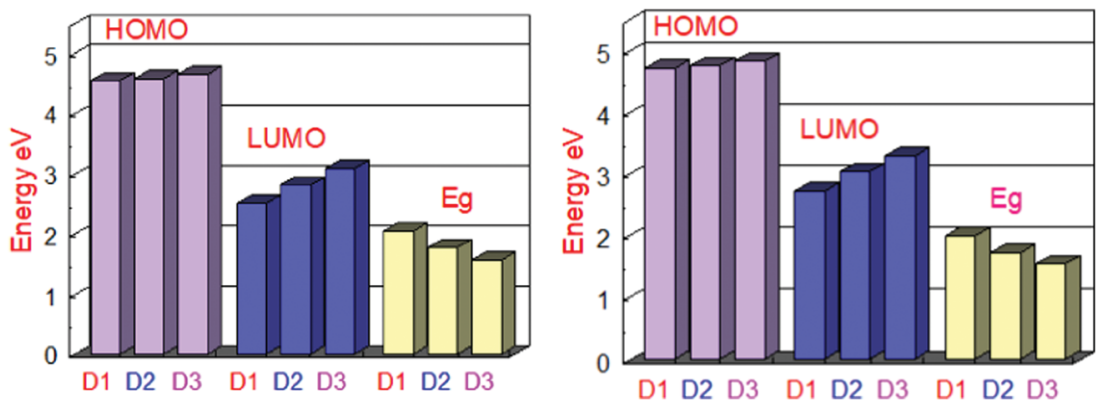

Fig. 3 Orbital energy representation of the designed molecules D1-D3 and their energy band gaps in the gas phase (left) and the solvent (right) 
Table 3 Computed maximum absorption wavelengths $\left(\lambda_{\max }\right)$, excitation energies $\left(E_{\mathrm{x}}\right)$, oscillator strengths $\left(f_{\mathrm{os}}\right)$, major molecular orbital assignments of reference $R$ and the designed molecules (D1-D4) in the gas phase at the TD-DFT/B3LYP/6-31G level of theory

\begin{tabular}{|c|c|c|c|c|c|}
\hline Molecules & State & $\begin{array}{l}\lambda_{\max } \\
(\mathrm{nm})\end{array}$ & $\begin{array}{l}f_{\text {os }} \\
\text { (a.u.) }\end{array}$ & $E_{\mathrm{x}}(\mathrm{eV})$ & Major MO assignments \\
\hline \multicolumn{6}{|l|}{ Gas } \\
\hline \multirow[t]{3}{*}{ D1 } & S1 & 738.46 & 0.4019 & 1.6790 & HOMO $\rightarrow$ LUMO(99\%) \\
\hline & S2 & 622.19 & 0.0057 & 1.9928 & HOMO-1 $\rightarrow$ LUMO(99\%) \\
\hline & S3 & 431.49 & 0.0171 & 2.8734 & HOMO-2 $\rightarrow$ LUMO $(99 \%)$ \\
\hline D2 & S1 & 852.24 & 0.4632 & 1.4548 & HOMO $\rightarrow$ LUMO(97\%) \\
\hline \multirow[t]{2}{*}{ D3 } & & 674.15 & 0.0600 & 1.8391 & HOMO-1 $\rightarrow$ LUMO(100\%) \\
\hline & & 460.50 & 0.0110 & 2.6924 & HOMO-2 $\rightarrow$ LUMO(99\%) \\
\hline \multirow[t]{3}{*}{ D3 } & S1 & 964.01 & 0.5398 & 1.2861 & HOMO $\rightarrow$ LUMO(99\%) \\
\hline & S2 & 731.76 & 0.0266 & 1.6943 & HOMO-1 $\rightarrow$ LUMO(99\%) \\
\hline & S3 & 632.10 & 0.0014 & 1.9615 & HOMO-2 $\rightarrow$ LUMO $(99 \%)$ \\
\hline \multicolumn{6}{|c|}{ Chlorobenzene } \\
\hline \multirow[t]{3}{*}{ D1 } & S1 & 777.58 & 0.4314 & 1.5945 & HOMO $\rightarrow$ LUMO(98\%) \\
\hline & S2 & 649.19 & 0.0062 & 1.9098 & HOMO-1 $\rightarrow$ LUMO(99\%) \\
\hline & S3 & 444.25 & 0.0196 & 2.8162 & HOMO $-2 \rightarrow$ LUMO(\%)(99\%) \\
\hline D2 & S1 & 908.01 & 0.5414 & 1.3655 & HOMO $\rightarrow$ LUMO $(98 \%)$ \\
\hline \multirow[t]{2}{*}{ D3 } & $\mathrm{S} 2$ & 705.78 & 0.0139 & 1.7567 & HOMO-1 $\rightarrow$ LUMO(100\%) \\
\hline & S3 & 469.97 & 0.0139 & 2.6382 & HOMO-2 $\rightarrow$ LUMO $(100 \%)$ \\
\hline \multirow[t]{5}{*}{ D3 } & S1 & 1017.00 & 0.6657 & 1.2191 & HOMO $\rightarrow$ LUMO(13\%) \\
\hline & S2 & 756.31 & 0.0379 & 1.6393 & HOMO-1 $\rightarrow$ LUMO(13\%) \\
\hline & S3 & 580.43 & 0.0010 & 2.1361 & HOMO-5 $\rightarrow$ LUMO(15\%) \\
\hline & & & & & HOMO-4 $\rightarrow$ LUMO(70\%) \\
\hline & & & & & HOMO-3 LUMO(99\%) \\
\hline
\end{tabular}

$([1,2,5]$ thiadiazolo[3,4- $d]$ pyridazine). D3 $>\mathrm{D} 2>\mathrm{D} 1$ is the decreasing order of all molecules' maximum values. This order corresponds to the reverse order of the HOMO-LUMO energy gap: D1 > D2 > D3, implying that designing and developing high-performance molecules for solar cells is exciting and appealing.

Changing the acceptor unit in the designed molecules is a great way to tune the photophysical properties and improve the photovoltaic properties. As a result, the newly developed molecules D1-D3 exhibit enhanced optoelectronic or photovoltaic properties. The excitation energy is vital for OSCs to improve the photovoltaic or optoelectronic properties. Our developed molecules have low excitation energy, making them ideal for photovoltaic or optoelectronic applications. Better charge transfer and a higher power conversion efficiency (PCE) in OSCs are observed in the designed molecules with a lower excitation energy and vice versa. The decreasing orders of excitation energy for all the proposed molecules in the gas and solvent phases are D1 $>$ D2 $>$ D3 and D1 $>$ D2 $>$ D3, respectively. Tables 2 and 3 show the computed max values, excitation energies $\left(E_{\mathrm{x}}\right)$, oscillator strengths $\left(f_{\mathrm{os}}\right)$, and significant molecular orbital assignments in the solvent and gas phases. Table 3 shows the important molecular orbital assignments or transitions that arose from HOMO-LUMO (99\%) across all the compounds D1-D3 in both gas and solvent phases. This is also a property of the designed molecules with enhanced optoelectronic capabilities. The simulated absorption spectra of molecules D1-D3 produced in gas and chloroform solvent phases are shown in Fig. 4 and 5, which indicate

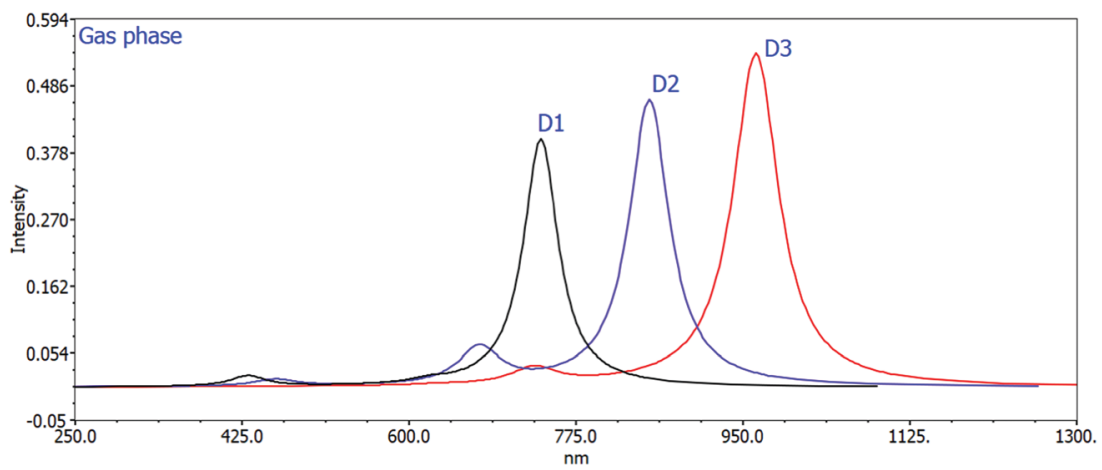

Fig. 4 Calculated UV spectra of D1, D2, and D3 using the TD-DFT/B3LYP/6-31G method in the gas phase.

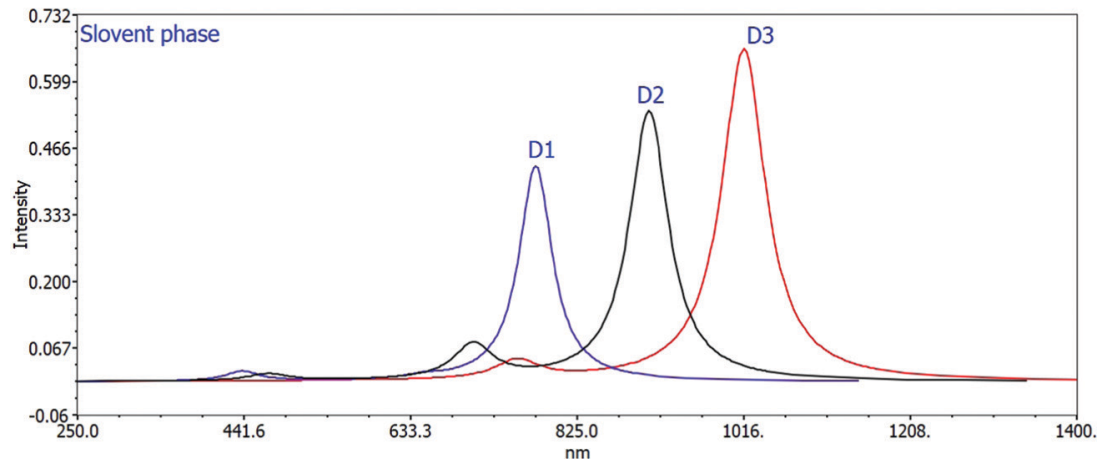

Fig. 5 Calculated UV spectra of D1, D2, and D3 using the TD-DFT/B3LYP/6-31G method in the solvent phase. 

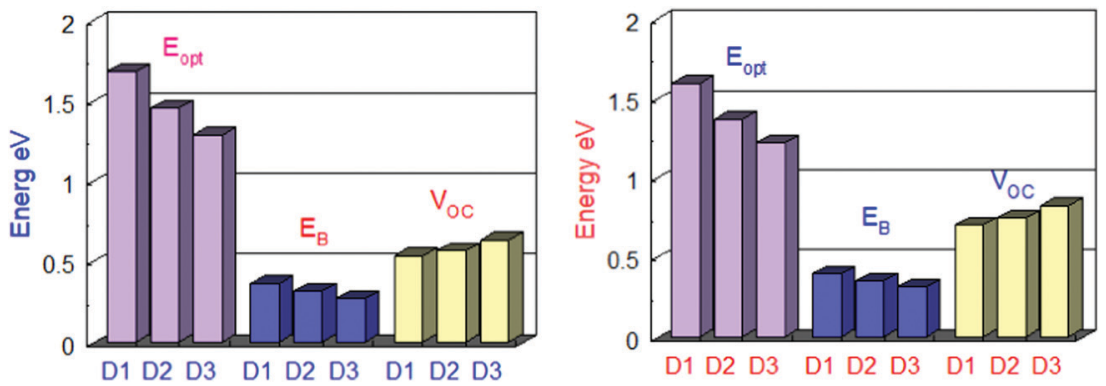

Fig. 6 Comparison between $E_{\mathrm{g}}, E_{\mathrm{opt}}$ and $E_{\mathrm{b}}$ of all the designed molecules in the gas phase (left) and in solvent (right).

the more prominent and more intense secondary absorption peak for each molecule. It is evident from the results in Tables 2 and 3 that end-capped unit modification has a significant impact on the max values of the designed molecules (D1-D3), resulting in a redshift in the UV/visible spectrum with a lower excitation energy and a 99 percent transition from the HOMO to the LUMO. As a result, the molecules (D1-D3) have exceptional optoelectronic capabilities.

\subsection{Open circuit voltage $\left(V_{\mathrm{OC}}\right)$}

The open-circuit voltage $\left(V_{\mathrm{OC}}\right)$ is also used to estimate organic solar cell performance. The efficiency of solar devices is proportional to the amount of $V_{\mathrm{OC}}$ produced. The $V_{\mathrm{OC}}$ measures the highest quantity of voltages or currents attained by photovoltaic devices in the zero current stage. $V_{\mathrm{OC}}$ with a high value improves the fill factor $(\mathrm{FF})$ of OSC devices, a significant factor in their efficiency. ${ }^{43}$ In photovoltaic devices, the open circuit voltage is influenced by photo-generated current and saturation voltage, favouring recombination. The LUMO energy of the acceptor and the HOMO energy of the donor molecules are directly related to the open-circuit voltage $V_{\mathrm{OC}}$. We calculated the $V_{\mathrm{OC}}$ of reference $\mathrm{R}$ and built molecules using Scharber and his coworkers' algorithm. ${ }^{44}$

$$
V_{\mathrm{OC}}=\frac{1}{e}\left(\left|E_{\mathrm{HOMO}}^{\text {Donor }}\right|-\left|E_{\mathrm{LUMO}}^{\mathrm{PCBM}}\right|\right)-0.3
$$

The HOMO of donor molecules (D1-D3) and the LUMO of the standard acceptor polymeric material PC71BM $([6,6]$ phenyl-C71-butyric acid methyl ester) display energy values of $6.1 \mathrm{eV}$ and $3.7 \mathrm{eV}$, respectively. ${ }^{43}$ Concerning LUMO values of of PC71BM-HOMO, the $V_{\mathrm{OC}}$ values obtained from Eqn (1). The energy gaps between donors are depicted in Fig. 6 and Table 4 . In the gas and solvent phases, the open-circuit voltages $\left(V_{\mathrm{OC}}\right)$ are $0.53 \mathrm{~V}, 0.56 \mathrm{~V}, 0.63 \mathrm{~V}$ and $0.70,0.74,0.82 \mathrm{~V}$ for $\mathrm{D} 1, \mathrm{D} 2$, and $\mathrm{D} 3$, respectively. The $V_{\mathrm{OC}}$ value of the $\mathrm{D} 3$ donor molecule is the highest of all. The decreasing order of $V_{\mathrm{OC}}$ for the designed molecules in both stages is D3 > D2 > D1.

The $V_{\mathrm{OC}}$ is usually determined by the HOMO and LUMO levels of the donor and acceptor. The lower LUMO value of the PC71BM acceptor creates a higher open-circuit voltage, which improves the photovoltaic characteristics of OSCs. The lower LUMO of the PC71BM acceptor causes an increase in electron transport from the HOMO of the donor to the LUMO of the
Table 4 HOMO-LUMO energy gaps $\left(E_{\mathrm{g}}\right)$, first singlet excitation energies $\left(E_{\mathrm{opt}}\right)$ and exciton binding energies $\left(E_{\mathrm{b}}\right)$

\begin{tabular}{lllllll}
\hline Donor & HOMO & LUMO & $E_{\mathrm{g}}(\mathrm{eV})$ & $E_{\mathrm{opt}}(\mathrm{eV})$ & $E_{\mathrm{b}}(\mathrm{eV})$ & $V_{\mathrm{OC}}$ \\
\hline Gas & & & & & & \\
D1 & -4.5359 & -2.4948 & 2.0410 & 1.6792 & 0.3618 & 0.5359 \\
D2 & -4.5691 & -2.8012 & 1.7678 & 1.4550 & 0.3128 & 0.5691 \\
D3 & -4.6363 & -3.0823 & 1.5539 & 1.2863 & 0.2676 & 0.6363 \\
PC71BM & -6.1 & -3.7 & - & - & - & - \\
Chlorobenzene & & & & & \\
D1 & -2.7155 & 1.9895 & 1.9895 & 1.5947 & 0.3948 & 0.7051 \\
D2 & -3.0301 & 1.7169 & 1.7169 & 1.3656 & 0.3513 & 0.7470 \\
D3 & -3.2915 & 1.5321 & 1.5321 & 1.2193 & 0.3128 & 0.8237 \\
& & & & & & \\
\hline
\end{tabular}

PC71BM acceptor, improving the photovoltaic characteristics of OSCs. Furthermore, the $E_{\mathrm{g}}$ of the proposed donor and PC71BM acceptor molecules boosts the OSC PCE values. The orbital energies of D1-D3 donors concerning the PC71BM acceptor is shown. Table 4 shows that the LUMO of PC71BM is lower than that of donor molecules (D1, D2 and D3). Therefore, the designed donor molecules (D1, D2, and D3) are shifted to the acceptor PC71BM, which improves the photovoltaic characteristics of all these molecules.

\section{5. $\quad$ Exciton binding energies $\left(E_{\mathbf{b}}\right)$}

The exciton binding energy determines the charge separation rate and efficiency of OSCs. The exciton binding energy measures the interaction of coulombic forces between electrons and holes. If the exciton binding energy is larger, the electronhole coulombic interaction will be higher, and vice versa. The exciton binding energy is calculated using the $E_{\mathrm{g}}$ value and the initial singlet excitation energy $\left(E_{\text {opt }}\right){ }^{45}$ The exciton binding energy is computed theoretically using eqn (2):

$$
E_{\mathrm{B}}=E_{\mathrm{g}}-E_{\mathrm{pot}}
$$

Table 4 shows the exciton binding energies for the developed and reference molecules. More significant charge separation is observed if the exciton binding energy is lower. As a result, the D3 molecule has a more considerable charge dissociation than the D1, D2, and D3 molecules. The results in Table 4 reveal that the designed molecules have lower exciton binding energies and display more charge separation than the other donors. For all the proposed molecules D1-D3, the lower the exciton binding energy, the higher the current charge density $\left(J_{\mathrm{sc}}\right)$. 
The exciton binding energy decreases in the order of D3 $>$ D2 $>$ D1. Fig. 6 shows a detailed comparison of $E_{\mathrm{g}}, E_{\mathrm{opt}}$, and $E_{\mathrm{b}}$.

\subsection{Dipole moment}

The polarity of the designed molecules (D1-D3) in the chlorobenzene solvent is determined by measuring their dipole moments. The solubility of OSC materials is directly proportional to the dipole moment. The solubility of OSC materials increases as the dipole moment increases. ${ }^{46}$ OSC materials have a reduced dipole moment due to their symmetry, diminishing their solubility in polar liquids (chlorobenzene). Because of the increase in the dipole moment, increasing the number of polar atoms in the designed molecules will enhance their solubility. The charge transport phenomenon of OSC molecules is also influenced by the dipole moment; the more extensive the dipole moment, the greater the flow of electrons between the ground and excited states. Therefore, the polarity of the designed molecules may rise in excited states. However, this is just for nanoseconds until de-excitation occurs. Table 1 shows the computed dipole moment strength of all the molecules in the chloroform solvent (CPCM model), both designed and reference. In the chloroform solvent, the predicted dipole moment strength is more significant, whereas it is lower in the gaseous phase. Because of the polar atoms in their architectures, the proposed compounds D1-D3 have a higher solubility in the chloroform solvent. In the gas and solvent phases, the dipole moments of the proposed molecules are 4.3873, 4.5499, 3.0075 Debye and 5.5353, 5.6140, 3.9134 Debye, respectively. In both the gas and solvent phases, the solubility order of all the designed molecules in the ground state is D2 > D1 > D3.

\subsection{Molecular electrostatic potential map analysis}

The molecular electrostatic potential surface map (MEP) depicts three-dimensional molecular charge patterns. These maps show the variable charged areas and the deficient and rich electron sites of the D1-D3 molecules. Charge distribution data can be used to determine how the molecules interact with one another. The positive and negative electrostatic potential in molecules D1-D3 is located using MEP map surfaces. A distinct colour scale shows the negative and positive electrostatic potential of each MEP map surface. The negative extreme in Fig. 7 (Fig. S4, ESI $\dagger-$ in chlorobenzene) is red, while the positive extreme is blue. The red colour indicates the minimum electrostatic potential (rich electron density) with a negative electrostatic potential, which acts as an electrophilic assault area. The blue colour shows the highest electrostatic potential (a lower electron density) and functions as a nucleophilic attack area. ${ }^{42}$ It is fascinating to note that all the developed (D1-D3) molecules have the same molecular electrostatic potential (MEP) distribution pattern, indicating that new molecules for solar cells can be designed efficiently and effectively. The centre acceptor and bridge units of D1-D3 molecules have a negative electrostatic potential (blue colour), but the endcapped donor units have a positive electrostatic potential (green colour) (red colour). The D3 molecule has a more excellent negative charge distribution (negative electrostatic potential) than the other donors, as shown in Fig. 7.

\subsection{Density of states and overlap population density of states}

The density of states (DOS) is the number of various states that electrons will occupy at a given energy level, i.e. the number of electron states per unit volume per unit energy. A high DOS value indicates numerous forms accessible for energy levels. The DOS value of zero indicates that there are no states accessible for energy level occupation. Calculating the DOS allows one to identify the gap between energy bands and the general distribution of states as a function of energy. ${ }^{47}$ In the boundary region, neighbouring orbitals may have quasidegenerate energy levels. The HOMO and LUMO alone cannot
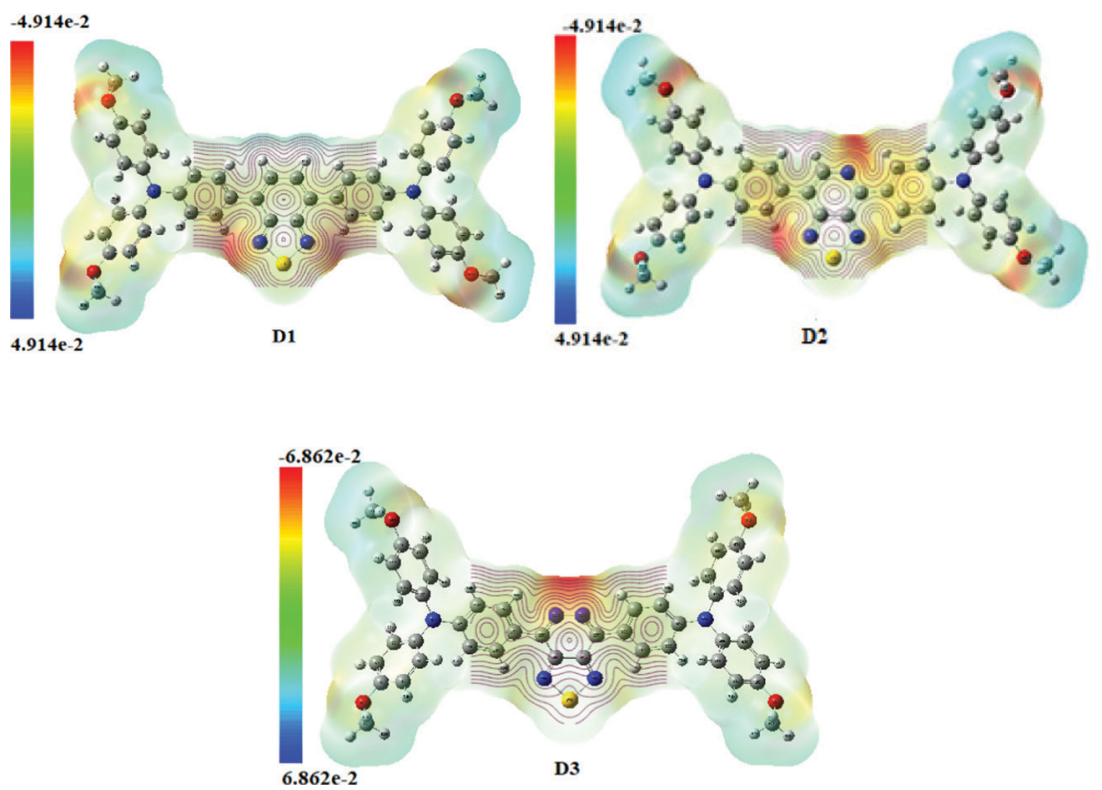

Fig. 7 MEP map representation of the designed molecules (D1-D3) in the gas phase. 


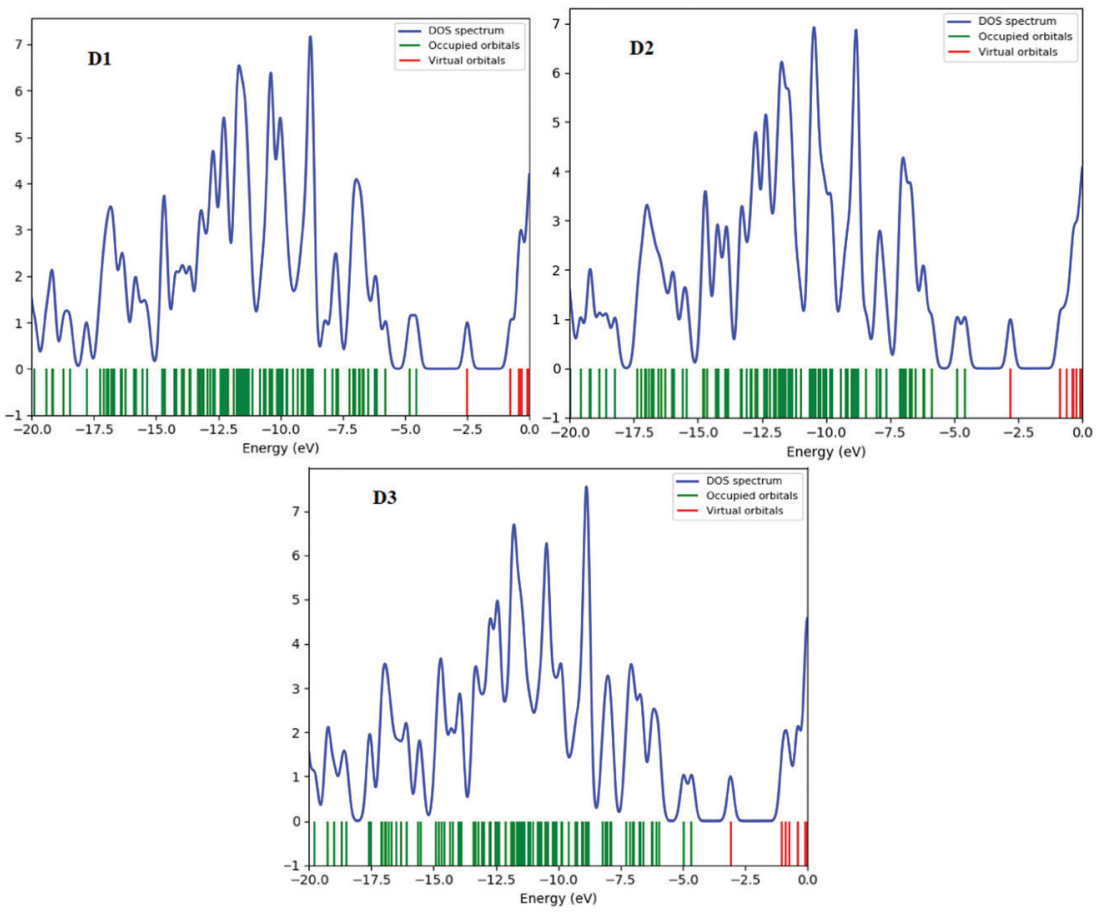

Fig. 8 DOS plots of the designed molecules D1-D3 in the gas phase.

offer a meaningful representation of frontier molecular orbitals. The density of states (DOS) is estimated for this purpose. Fig. 8 and (Fig. S5, ESI $\dagger$ - in chlorobenzene) show the DOS plots of D1-D3 molecules at the B3LYP/6-31G level of theory, respectively. Determining molecular orbital (MO) configurations is the most important use of density of states (DOS) charts.

Red, green, and blue lines represent the electronic distribution of donor and acceptor bridge groups in the DOS plots of the designed D1-D3 molecules, respectively. The DOS plot presents the orbital properties at various energy levels. In the DOS plots, the effect of the bridge group on the distribution patterns of all the proposed molecules can be seen (Fig. 8). The donor groups contribute around 20 to $-0.0 \mathrm{eV}$ energy to the frontier molecular orbitals in the planned molecules D1, D3, and D4. The negative and positive energies represent the HOMO and LUMO orbital energies along the $x$-axis, respectively; the HOMO-LUMO energy gap represents the distance between positive and negative energies.

\section{Conclusion}

State-of-the-art quantum chemical approaches were employed to investigate the effect of end-capped unit modifications of the designed TPA (triphenylamine) based materials (D1-D3) on the structure-activity, charge transfer and optoelectronic properties. These molecules have shown red-shifted and broader absorption spectra in the range of 738.46-964.01 $\mathrm{nm}$ in the gas phase and in the range of 777.58-1017.00 nm in chlorobenzene; the lower binding energies $\left(E_{\mathrm{b}}\right)$ of D1-D3 result from higher exciton dissociation at excited states. The optoelectronic properties of D3 are superior to those of D1 and D2 due to better charge transfer and extended conjugation of the $[1,2,5]$ thiadiazolo[3,4- $d]$ pyridazine acceptor. The D3 molecule with higher charge transferability is the highly recommended material for OSC applications, bearing the lowest $E_{\mathrm{g}}(1.5321 \mathrm{eV})$, excitation energy $(1.2193 \mathrm{eV})$, and $E_{\mathrm{b}}(0.3128 \mathrm{eV})$, highest $\lambda_{\max }$ values of $964.01 \mathrm{~nm}$ (in gas) and $1017.00 \mathrm{~nm}$ (in chlorobenzene), and a higher $V_{\mathrm{OC}}(0.8237 \mathrm{~V})$ regarding $\mathrm{LUMO}_{\mathrm{PC} 71 \mathrm{BM}}-\mathrm{HOMO}_{\text {Donor }}$. Thus, these acceptor unit modification is an effective strategy to design efficient and effective next-generation OSC devices. Therefore, D1-D3 molecules are highly recommended to experimentalists to develop highly efficient solar cell devices.

\section{Conflicts of interest}

There are no conflicts to declare.

\section{References}

1 L. Duan, et al., Dopant-free X-shaped D-A type holetransporting materials for $\mathrm{p}-\mathrm{i}-\mathrm{n}$ perovskite solar cells, Dyes Pigm., 2020, 178, 108334.

2 G. E. Alvarez, M. G. Marcovecchio and P. A. Aguirre, Optimization of the integration among traditional fossil fuels, clean energies, renewable sources, and energy storages: An MILP model for the coupled electric power, hydraulic, and natural gas systems, Comput. Ind. Eng., 2020, 139, 106141.

3 M. Shoaib, et al., Assessment of wind energy potential using wind energy conversion system, J. Cleaner Prod., 2019, 216, 346-360.

4 U. K. Pata and M. Aydin, Testing the EKC hypothesis for the top six hydropower energy-consuming countries: Evidence 
from Fourier bootstrap ARDL procedure, J. Cleaner Prod., 2020, 264, 121699.

5 S. N. B. Villadsen, et al., The potential of biogas; the solution to energy storage, ChemSusChem, 2019, 12(10), 2147-2153.

6 C. Scherelis, et al., Dataset for concurrent echo sounder and ADCP measurements at a tidal energy candidate site in Australia, Data. Br., 2020, 31, 105873.

7 M. Adnan and J. K. Lee, Highly efficient planar heterojunction perovskite solar cells with sequentially dip-coated deposited perovskite layers from a non-halide aqueous lead precursor, RSC Adv., 2020, 10(9), 5454-5461.

8 M. Adnan, Z. Irshad and J. K. Lee, Facile all-dip-coating deposition of highly efficient ( $\mathrm{CH} 3)^{3}$ NPbI $3-\mathrm{x} \quad \mathrm{Cl} x$ perovskite materials from aqueous non-halide lead precursor, RSC Adv., 2020, 10(48), 29010-29017.

9 Z. Irshad, M. Adnan and J. K. Lee, Efficient planar heterojunction inverted perovskite solar cells with perovskite materials deposited using an aqueous non-halide lead precursor, Bull. Korean Chem. Soc., 2020, 41(9), 937-942.

$10 \mathrm{M}$. Adnan and J. K. Lee, All sequential dip-coating processed perovskite layers from an aqueous lead precursor for high efficiency perovskite solar cells, Sci. Rep., 2018, 8(1), 2168.

11 M. Naeem, et al., Tuning of optoelectronic properties of triphenylamines-based donor materials for organic solar cells, J. Theor. Comput. Chem., 2019, 18(7), 1950036.

12 M. Adnan, et al., Fine tuning the optoelectronic properties of triphenylamine based donor molecules for organic solar cells, Z. Phys. Chem., 2017, 231(6), 1127-1139.

13 Y. Sun, et al., Solution-processed small-molecule solar cells with $6.7 \%$ efficiency, Nat. Mater., 2012, 11(1), 44-48.

$14 \mathrm{~J}$. Roncali, et al., Molecular and supramolecular engineering of $\pi$-conjugated systems for photovoltaic conversion, Thin Solid Films, 2006, 511-512, 567-575.

$15 \mathrm{X}$. Sun, et al., X-shaped oligothiophenes as a new class of electron donors for bulk-heterojunction solar cells, J. Phys. Chem. B, 2006, 110(15), 7702-7707.

$16 \mathrm{~J}$. Zhou, et al., Solution-processed and high-performance organic solar cells using small molecules with a benzodithiophene unit, J. Am. Chem. Soc., 2013, 135(23), 8484-8487.

17 Q. Zhang, et al., Small-molecule solar cells with efficiency over 9\%, Nat. Photonics, 2015, 9(1), 35-41.

18 T. Jadoon, et al., Silver clusters tune up electronic properties of graphene nanoflakes: A comprehensive theoretical study, J. Mol. Liq., 2020, 297, 111902.

19 R. Hussain, et al., Molecular engineering of A-D-C-D-A configured small molecular acceptors (SMAs) with promising photovoltaic properties for high-efficiency fullerene-free organic solar cells, Opt. Quantum Electron., 2020, 52(8), 1-20.

20 M. Bilal Ahmed Siddique, et al., Designing triphenylamineconfigured donor materials with promising photovoltaic properties for highly efficient organic solar cells, ChemistrySelect, 2020, 5(25), 7358-7369.

21 M. Y. Mehboob, et al., Designing $N$-phenylaniline-triazol configured donor materials with promising optoelectronic properties for high-efficiency solar cells, Comput. Theor. Chem., 2020, 1186, 112908.
22 Z. Afzal, et al., Designing indenothiophene-based acceptor materials with efficient photovoltaic parameters for fullerene-free organic solar cells, J. Mol. Model., 2020, 26(6), 137.

23 M. J. Frisch, et al., Gaussian 09, Revision A.02, Gaussian, Inc., Wallingford, CT, 2009.

24 E. Frisch, et al., GaussView, Version 5.0.8, Gaussian, Inc., Wallingford, 2009.

25 A. Savin, in Density Functional Methods in Chemistry, ed. J. K. LLabanowski, J. W. Andzelm, Springer-Velrag, New York, 1991, pp. 213-230.

26 P. M. W. Gill, B. G. Johnson, J. A. Pople and M. Frisch, J. Chem. Phys. Lett., 1992, 197, 499.

27 R. G. Parr and W. Yang, Density-Functional Theory of Atoms and Molecules, Oxford University Press, Oxford, 1989.

28 A. D. Becke, Density-functional thermochemistry. III. The role of exact exchange, J. Chem. Phys., 1993, 98(7), 5648-5652.

29 M. J. Frisch, J. A. Pople and J. S. Binkley, Self-consistent molecular orbital methods 25. Supplementary functions for Gaussian basis sets, J. Chem. Phys., 1984, 80(7), 3265-3269.

30 R. Reslan, et al., J. Chem. Phys., 2012, 137, 22.

31 R. Bauernschmitt and R. Ahlrichs, Treatment of electronic excitations within the adiabatic approximation of time dependent density functional theory, Chem. Phys. Lett., 1996, 256(4-5), 454-464.

32 F. Furche and R. Ahlrichs, Adiabatic time-dependent density functional methods for excited state properties, J. Chem. Phys., 2002, 117(16), 7433-7447.

$33 \mathrm{P}$. Su and $\mathrm{H}$. Li, Continuous and smooth potential energy surface for conductorlike screening solvation model using fixed points with variable areas, J. Chem. Phys., 2009, 130(7), 074109.

34 N. M. O’Boyle, A. L. Tenderholt and K. M. Langner, cclib: A library for package-independent computational chemistry algorithms, J. Comput. Chem., 2008, 29(5), 839-845.

$35 \mathrm{H}$. Tan, et al., Highly efficient hybrid polymer and amorphous silicon multijunction solar cells with effective optical management, Adv. Mater., 2016, 28(11), 2170-2177.

36 A. Patra, et al., Metal free conducting pedos, Pedot, and their analogues via an unusual bromine-catalyzed polymerization, Macromolecules, 2015, 48(24), 8760-8764.

37 A. Patra, M. Bendikov and S. Chand, Poly(3,4-Ethylenedioxyselenophene) and its derivatives: Novel organic electronic materials, Acc. Chem. Res., 2014, 47(5), 1465-1474.

38 R. A. Shehzad, et al., Designing of benzothiazole based nonfullerene acceptor (NFA) molecules for highly efficient organic solar cells, Comput. Theor. Chem., 2020, 1181, 112833.

39 N. Ohta, K. Yamashita and A. Muraoka, Mechanism of charge transfer and separation in polymer/nonfullerene acceptor organic solar cells, Bull. Am. Phys. Soc., 2020, 65, 7148.

40 G. Deogratias, N. Seriani, T. Pogrebnaya and A. Pogrebnoi, Tuning optoelectronic properties of triphenylamine based dyes through variation of pi-conjugated units and anchoring groups: A DFT/TD-DFT investigation, J. Mol. Graphics Modell., 2020, 94, 107480. 
41 J. S. Murray and P. Politzer, Molecular electrostatic potentials and noncovalent interactions, Wiley Interdisciplinary Reviews: Computational Molecular Science, Blackwell Publishing, Inc., 2017, vol. 7 (6), 01-Nov.

42 M. Adnan, et al., Efficient synthesis and characterization of solvatochromic fluorophore, Bull. Korean Chem. Soc., 2017, 38(9), 1052-1057.

$43 \mathrm{~J}$. Zhao, et al., High-efficiency non-fullerene organic solar cells enabled by a difluorobenzothiadiazole-based donor polymer combined with a properly matched small molecule acceptor, Energy Environ. Sci., 2015, 8(2), $520-525$.
44 M. C. Scharber, et al., Design rules for donors in bulkheterojunction solar cells - Towards 10\% energy-conversion efficiency, Adv. Mater., 2006, 18(6), 789-794.

45 S. Hussain, et al., Zinc-doped boron phosphide nanocluster as efficient sensor for SO2, J. Chem., 2020, 2020, 1-12.

46 N. Qiu, et al., A new nonfullerene electron acceptor with a ladder type backbone for high-performance organic solar cells, Adv. Mater., 2017, 29(6), 1604964.

47 P. Goszczycki, et al., Synthesis, crystal structures, and optical properties of the $\pi-\pi$ interacting pyrrolo[2,3- $b]$ quinoxaline derivatives containing 2-thienyl substituent, J. Mol. Struct., 2017, 1146, 337-346. 